\title{
DETECTION OF ONE-, TWO-, AND THREE-DIMENSIONAL MARKOV CONSTRAINTS IN VISUAL DISPLAYS II: MULTI- STATE DISPLAYS
}

\author{
Irwin POLLACK* \\ Mental Health Research Inst., University of Michigan, Ann Arbor, Mich. 48104, U.S.A.
}

Received January 1975

\begin{abstract}
The study of the depth of visual information processing is here extended to multistate displays. Two classes of variables are distinguished: display variables which may either be fixed at a single level or varied over its possible levels, e.g., a numeric character, its brightness, and its orientation; and spatial-temporal variables which assume all possible states within each display, e.g., the $x$ - and $y$-coordinates of the display, and the time-coordinate, $t$, representing successive frames of the display. Information was encoded in terms of constraints upon combinations of variables. Excellent discrimination is achieved for detecting constraints among two, but not three, display variables; or for detecting constraints among one, but not two, display variables and up to three spatial-temporal variables. Comparisons are made with previous tests of the depth of visual information processing with binary-coded materials within the spatial-temporal microstructure of the display.
\end{abstract}

\section{Introduction}

In a previous report, the depth of visual information processing was explored where informational constraints were encoded within the spatial microstructure of the displays. The display elements were individual dots within $18 \times 18$ matrices, where each element could assume one of two states: the absence or presence of a dot. A 'perceptual' solution to the discovery of informational constraints was presumably dominant since information was encoded in terms of the relations among the individual dot elements and because displays were

\footnotetext{
* This research was supported in part by a grant of the National Sciences Foundation. The author is indebted to Linda Matich for supervision of the tests and to Thomas Frohlich for the experimental program.
} 
briefly presented. Excellent discrimination was shown for one-dimensional constraints in terms of the spatial dimensions, $X$ and $Y$, and in terms of the temporal dimension, $T$; and for two-dimensional constraints in terms of $X Y, X T$ and $Y T$; but not for the three-dimensional combination, $X Y T$ (Pollack 1971).

The present study sought to explore the detection of multi-variate informational constraints when the individual variables could take on more than two states. To permit a 'cognitive or intellectual' solution to the discovery of informational constraints, numeric characters were employed as the display elements. Following Garner and Gottwald (1969), it is also assumed that 'perceptual' processing is primarily characteristic of rapid rates of display presentation; and that 'cognitive' processing is primarily characteristic of slow rates of display presentation.

\section{Method}

\subsection{Apparatus}

A PDP-9 computer was programmed to produce sequences of displays upon an $8 \mathrm{~cm} \times$ $8 \mathrm{~cm}$ surface of a Tcktronix 602 display, equipped with a fast $P-15$ phosphor.

\subsection{Variables}

It is convenient to distinguish between two classes of display variables which may be fixed at a single level or varied over eight levels: $C$ (a numeric character from 1 to 8 ), $B$ (its brightness), $O$ (its orientation); and spatial-temporal variables which always existed at all eight levels: $X$ (the $x$-coordinate or columns of an $8 \times 8$ matrix), $Y$ (the $y$-coordinate or rows of an $8 \times 8$ matrix), and $T$ (the temporal order of a sequence of successive displays).

\subsection{Procedure: general}

Upon a single observation a sequence of 8 successive display-fields was presented. The sequences were drawn from distributions with identical statistical parameters. In one case, a further informational constraint was imposed upon the selection of variables; in the other, the constraint was missing. The combination of the presence and absence of a constraint defined a paired contrast. A block of 24 successive observations employed a single paired contrast without a change of variables. The observer was instructed to press one of two response buttons indicating whether the display-sequence was (a) relatively random, or was (b) relatively organized (see below). Immediate feedback was given after each response. 
EXAMPLES OF 1-D, 2-D AND 3-D VISUAL CONSTRAINTS
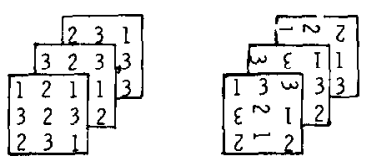

random constraint
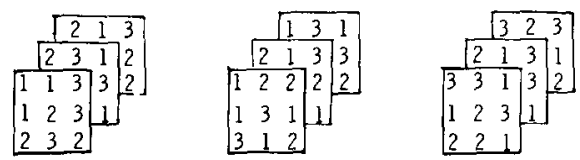

complete constraint

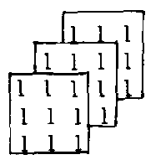

A. Character alone

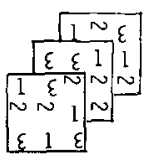
B. Character and orientation.

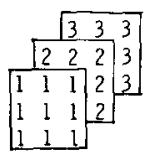
D. Character and
Time
C. Character $x$-Coordinate

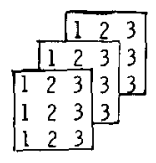

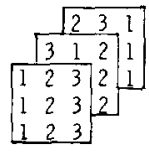

E. Character
-Coordinate
and
Time

Fig. 1. Illustration of multi-variate constraints (see text, section 2.5).

\subsection{Informational restrictions}

Typically, a random constraint was paired against a comf'ete informational constraint. The method, however, is general and a contrast could consist of any pairing of constraints. A given display variable may be subjected to an informational constraint, may vary extraneously at random, or may be held constant at one of its possible states. Any spatial-temporal variable can be combined with one or more display variables within an informational constraint.

\subsection{Examples of informational constraints}

\subsubsection{One-dimensional constraints}

One dimensional (1-D) constraints were restricted to display variables, and were reflected in terms of the relative frequency of occurrence of the restricted variable. Fig. 1 illustrates several contraints involving the display variable of the numeric Character. Within each column of fig. 1 three successive display-fields with three states per variable are illustrated. The displays reflect either a random constraint (top half) or the indicated complete informational constraint (bottom half). For display purposes, the second and third display-fields are drawn as partially occluded, and only three states per variable are represented. In the actual tests, the centers of characters overlapped on successive display fields, and eight states per variable were employed.

For the 1-D constraint on Character of fig. $1 \mathrm{~A}$, for example, a random constraint is reflected by the equally likely distribution of all characters within each display-field; a complete constraint was reflected by the entire display consisting of a single character; a partial constraint was reflected by a relatively greater frequency of one of the characters, with the remaining characters equally likely. Note that the complete constraint is recognizable either in terms of the uniformity over each of the displays (all 1's), or because the display is identical over successive display-fields. Similarly, a random, a complete, or a partial constraint could be 
reflected in the relatively greater frequency of one of 8 brightness levels, or in one of 8 orientations.

\subsubsection{Multi-dimensional constraints}

Constraints could be imposed upon combinations of more than one display variable; or between one or more display variables and a spatial-temporal variable.

In general, a constrain $\uparrow$ involving $n$-display variables employed a random distribution among $(n-1)$ display variables, and with the state of the $n$th variable determined the combination of the variables and a parity sum. For example, a random two-dimensional (2-D) constraint upon Character and Orientation, illustrated in fig. $1 \mathrm{~B}$, is reflected by each possible character occurring equally often with each possible orientation; and a complete constraint is reflected by each of the possible characters occurring equally often, but a unique character is associated with a unique orientation level (all 1's are upright, all 3's are inverted, etc.). Even in the case of a perfect constraint between two display variables, the spatial relations within a display-field or across successive display-fields are random.

By contrast, the perfect constraint between one display variable and one spatial-temporal variable, as illustrated in fig. $1 \mathrm{C}$, leads to a uniform display across successive display-fields, or as illustrated in fig. 1D, leads to uniform display-fields which change in time.

Although figs. $1 \mathrm{~B}, 1 \mathrm{C}$, and $1 \mathrm{D}$ each represent a constraint upon the combination of two variables, there is a profound difference in the apparent degree of scrambling of two display variables (fig. 1B) and one display plus one spatial-temporal variable (fig. 1C and fig. 1D). In each case, each of the possible states is either equally likely (display variables) or occurs equally often (spatial-temporal variables). In each case, only the combination of the variables is restricted. As expected, large differences in detection performance were found, depending upon the type of variable employed to illustrate the constraints.

A three-dimensional constraint is illustrated in fig. $1 \mathrm{E}$ for a single display variable and two spatial-temporal variables. In the example illustrated, a perfect constraint is reflected by successive rows sweeping over the displays. A perfect four-dimensional constraint (not illustrated) in $C X Y T$ is reflected by diagonals of like characters within successive display-fields sweeping over the display.

\subsection{Method of specifying informational constraints}

The measure of the informational constraint is the probability of a zero aimed for parity sum among the $n$-variables. With a random constraint, all possible parity sums are equally likely. Thus, the $n$th variable is random, given a particular pattern of the other $(n-1)$ variables. With a partial constraint, one parity sum is more likely than the remaining equally likely parity sums. Thus, one state of the $n$th variable is more likely than others, given the particular pattern of the other $(n-1)$ variables.

With three states per variable, for example, a zero parity sum yields a complete informational restraint by pairing states 0,1 and 2 of one variable with states 0,2 and 1 of the second variable $[0+0=0,1+2=3=0(\bmod 3)]$. Unless specified otherwise, a 'dummy' variable was inserted so that, with a complete constraint, states 0,1 and 2 of one variable were paired with states 0,1 and 2 of the second variable. 


\subsection{Experimental details}

Testing was carried out in a dimly lit room. The observer adjusted the viewing distance to the display for maximum comfort, with most observers employing a distance of about $70 \mathrm{~cm}$. Unless specified otherwise, each display field consisted of 64 characters arranged in an $8 \times 8$ matrix without grid lines. Display duration was controlled in integral multiples of 28.6 msec. The brightness levels were obtained by equal-voltage differences applied to a digital-to-analog converter. Phenomenally, there was little differentiation among the four highest brightness levels, but clearer differentiation was available among the four lower brightness levels. Each of the individual characters was plotted upon a $5 \times 7$ grid. The distance between successive characters (center-to-center) was approximately $1 \mathrm{~cm}$. Successive steps in Orientation were $45^{\circ}$.

\subsection{Procedure: detailed}

- Two series of tests were run. All $S$ s had intensive experience in a wide variety of auditory and visual psychophysical studies. In Series I, $6 \mathrm{~S}$ s contributed two blocks of 24 observations each under each of 52 experimental conditions. Successive blocks employed successively weaker constraints for a given experimental condition. The duration between successive display-fields was either 29 or 114 msec. In Series II, 3 of the previous $6 S$ s contributed $2-3$ blocks of 24 observations under each of 100 conditions. Typically, a given experimental condition was represented over three successive blocks at a constant constraint level, but scrambled with respect to durations of 29,114 and $458 \mathrm{msec}$, respectively.

\subsection{Additional tests}

Two changes were introduced into the basic paradigm in order to study the temporal features of the displays more closely. In one set of tests, only the first display of the sequence of eight displays was flashed. In another set, blank fields were introduced between successive displays so that the on-time of the display was not completely confounded with the time between successive displays. Each of 3 experienced $S$ s contributed 2-3 blocks of 24 observations under each of 100 conditions.

\section{Results}

\subsection{Incidental observations}

Investigators in the field of concept identification typically differentiate between concept discovery and concept discrimination. For example, $C B X T$ refers to a restriction among combinations of variables in which, when the character 1 appears in the first column of the first of eight display fields, it is restricted to brightness level one. It is highly unlikely that an observer would discover that relationship without specific prompting. Moreover, even with specific prompting, it is unclear whether such complex combinations can be detected in a quickly flashing sequence of displays. Unless noted otherwise, all tests were of the concept discovery type, although complete informational constraints typically preceded partial con- 
straints in order to expedite the process of concept discovery. Within such limits, the observers found combinations of three display variables or two display variables plus one or more spatial-temporal variables to be extremely difficult.

The phenomenal appearance of the sequences changes radically with the interval between successive displays. At 35 display-frames per sec, judgements related to frame position are often based upon apparent brightness (later frames being brighter than earlier frames) or upon the uniformity of brightness and rotation. At slower rates of presentations, the individual characters can often be read and their associated brightness and orientation noted.

\subsection{Series $I$}

Table 1 summarizes the results of Series I. The first column lists the display duration for successive display frames: either $29 \mathrm{msec} /$ frame (or 35 frames per sec) or $114 \mathrm{msec} /$ frame (or 9 frames per sec); the second column lists the probability of a specific parity sum; the third column lists the combination of display variables paired with the spatial-temporal variables heading the next six columns. The tabular entries represent the percentage of correct twoalternative responses. Chance response is represented by $50 \%$ correct.

The constraint of a single display variable, here $C$ or character, with $0,1,2$ or 3 spatial-temporal variables can be detected even at the faster rate of presentation. At the faster rate of presentation, even with the most extreme parity sum, two- and three-variable combinations yield nearly chance performance, except for two-variable combinations with no spatialtemporal constraint.

At the slower rate of presentation, two-variable combinations are difficult to discern with a partial informational constraint.

\subsection{Series $I I$}

The observers were specifically instructed that they might seek out constraints such as 'bright 1's,' 'upright 1's,' 'bright uprights' in conjunction with spatial-temporal constraints.

Table 1

Percent correct observations for detection of constraints among combinations of display and spatial-temporal variables.

\begin{tabular}{lllllllll}
\hline $\begin{array}{l}\text { Display } \\
\text { duration } \\
\text { msec }\end{array}$ & & $\begin{array}{l}\text { Display } \\
\text { variables }\end{array}$ & \multicolumn{2}{l}{ Spatial-temporal variables } & & \\
\hline & $P(P S)$ & & None & $X$ & $T$ & $X Y$ & $X T$ & $X Y T$ \\
\hline 29 & 1.0 & $C$ & 98 & 98 & 92 & 93 & 90 & $84 \%$ \\
& & $C O$ & 69 & 57 & 50 & 56 & 46 & 55 \\
& & $C B O$ & 55 & 51 & 52 & 55 & 51 & 53 \\
114 & 0.8 & $C O$ & 64 & 54 & 51 & 56 & 52 & 48 \\
& 0.8 & $C$ & & 92 & 93 & 81 & 94 & 91 \\
& 0.6 & $C$ & & 79 & 90 & 74 & 74 & 83 \\
& 0.4 & $C$ & 80 & 67 & 62 & 63 & 62 & 48 \\
\hline
\end{tabular}


Table 2

Percent correct identifications of combinations of display and spatial-temporal variables.

\begin{tabular}{|c|c|c|c|c|c|c|c|c|c|c|c|c|}
\hline \multirow{2}{*}{$\begin{array}{l}\text { Display } \\
\text { duration } \\
\text { msec. }\end{array}$} & \multirow[b]{2}{*}{$P(P S)$} & \multirow{2}{*}{$\begin{array}{l}\text { Display } \\
\text { variables }\end{array}$} & \multicolumn{6}{|c|}{ Spatial-temporal variables } & \multicolumn{4}{|c|}{ Averages } \\
\hline & & & None & $X$ & IT & $X Y$ & $X T$ & $X Y T$ & $C$ & $\phi$ & $B$ & Aver \\
\hline \multirow[t]{4}{*}{29} & $0.75^{\mathrm{a}}$ & $C$ & 100 & 99 & 96 & 89 & 91 & 85 & 93 & & & \\
\hline & & $\phi$ & 99 & 99 & 98 & 98 & 88 & 83 & & 94 & & \\
\hline & & $B$ & 87 & 99 & 92 & 99 & 90 & 98 & & & 94 & \\
\hline & & Aver. & 95 & 99 & 95 & 95 & 90 & 89 & & & & 94 \\
\hline \multirow[t]{4}{*}{114} & & $C$ & 99 & 98 & 99 & 98 & 99 & 95 & 98 & & & \\
\hline & & $\phi$ & 99 & 98 & 99 & 94 & 99 & 84 & & 96 & & \\
\hline & & $B$ & 93 & 98 & 93 & 94 & 98 & 99 & & & 96 & \\
\hline & & Aver. & 97 & 98 & 97 & 95 & 99 & 93 & & & & 97 \\
\hline \multirow[t]{5}{*}{458} & & $C$ & 100 & 99 & 100 & 98 & 99 & 98 & 99 & & & \\
\hline & & $\phi$ & 99 & 99 & 100 & 95 & 99 & $(84)$ & & 96 & & \\
\hline & & $B$ & 97 & 92 & 92 & 99 & 98 & 98 & & & 96 & \\
\hline & & Aver. & 99 & 97 & 97 & 97 & 99 & 93 & & & & 97 \\
\hline & & & & & & & & & $C \phi$ & $C B$ & $\phi_{B}$ & \\
\hline \multirow[t]{4}{*}{29} & $1.0^{*}$ & $C \varnothing$ & (64) & (57) & $(50)$ & $(56)$ & (46) & (55) & 55 & & & \\
\hline & & $C B$ & 67 & 60 & 66 & 60 & 46 & 50 & & 58 & & \\
\hline & & $\not B$ & 72 & 54 & 52 & 45 & 49 & $(50)$ & & & 54 & \\
\hline & & Aver. & 68 & 57 & 56 & 54 & 47 & 52 & & & & 56 \\
\hline \multirow[t]{4}{*}{114} & & $C \varnothing$ & 65 & 68 & 69 & 58 & 54 & 46 & 60 & & & \\
\hline & & $C B$ & 75 & 46 & 66 & 57 & 51 & 55 & & 58 & & \\
\hline & & $\phi B$ & 69 & 55 & 51 & 48 & 59 & (50) & & & 55 & \\
\hline & & Aver. & 70 & 56 & 62 & 54 & 55 & 50 & & & & 58 \\
\hline \multirow[t]{4}{*}{458} & & $C \phi$ & 80 & 74 & 82 & 60 & 62 & 51 & 68 & & & \\
\hline & & $C B$ & 84 & 56 & 68 & 56 & 53 & 51 & & 61 & & \\
\hline & & $\phi B$ & 64 & 59 & 51 & 53 & 56 & $(50)$ & & & 56 & \\
\hline & & Aver. & 76 & 63 & 67 & 56 & 57 & 51 & & & & 62 \\
\hline
\end{tabular}

* $P(P S)=0.75$ with 'none'.

Entries within parentheses are taken from other tests, or are estimated.

However, the specific constraints were not identified to the observers for specific observations.

The organization of table 2 follows that of table 1, with expansion to still longer presentation times. Constraints between a single display variable and up to three spatial-temporal variables can easily be discerned, as shown in the top half of table 2 . And, performance improves undramatically with longer presentation times.

With two display variables, presented in the lower half of table 2, performance is rarely substantially higher than chance, even with the selected group of observers and even with the general instructions to look for combinations of variables. 
Table 3 (see section 3.4)

Percent correct identifications of combinations of display and spatial temporal variables.

\begin{tabular}{|c|c|c|c|c|c|c|c|}
\hline & $C$ & $C X Y T$ & $C \phi$ & $C B$ & $\phi_{B}$ & $C \emptyset_{B}$ & Variables \\
\hline $\begin{array}{l}\text { Frames } \\
\text { on }- \text { off }\end{array}$ & 0.3 & 0.7 & 0.75 & 0.75 & 1.0 & 1.0 & $P(P S)$ \\
\hline $1-0$ & 86 & 75 & 68 & 70 & 72 & 58 & $\begin{array}{l}\text { aver. } \\
72\end{array}$ \\
\hline $1-1$ & 83 & 85 & 80 & 76 & 74 & 61 & 77 \\
\hline $2-0$ & 88 & 95 & 76 & 76 & 80 & 62 & 80 \\
\hline $1-15$ & 93 & 89 & 90 & 83 & 85 & 63 & 84 \\
\hline $2-14$ & 92 & 84 & 88 & 85 & 82 & 69 & 83 \\
\hline $16-0$ & 94 & 95 & 86 & 76 & 84 & 64 & 83 \\
\hline $1-s$ & 80 & 82 & 75 & 67 & 65 & 60 & 72 \\
\hline $2-s$ & 76 & 83 & 79 & 66 & 78 & 65 & 74 \\
\hline $16-s$ & 81 & 82 & 79 & 72 & 84 & 63 & 77 \\
\hline Aver. $s$ & 79 & 82 & 78 & 68 & 76 & 63 & \\
\hline Aver. $x-0$ & 89 & 88 & 77 & 74 & 79 & 61 & \\
\hline
\end{tabular}

$\mathrm{a}$ one frame $=28.6 \mathrm{msec}$

Table 4 (see section 3.5)

Percent correct identifications as a function of the control of non-constrained variables. ${ }^{a}$

\begin{tabular}{|c|c|c|c|c|c|c|c|c|}
\hline \multirow{3}{*}{$\begin{array}{l}\text { Variables: } \\
\text { Constrained } \\
\text { Extraneous } \\
\text { Slaved }\end{array}$} & \multicolumn{5}{|c|}{$C$ constrained, $P(P S)=0.3$} & \multicolumn{3}{|c|}{$C X Y T$ constrained, $P(P S)=0.7$} \\
\hline & - & $\phi$ & $B$ & $\phi, B$ & - & - & $\phi, B$ & - \\
\hline & - & -- & - & - & $\phi, B$ & - & - & $\phi, B$ \\
\hline \multicolumn{9}{|l|}{$\begin{array}{l}\text { Frames } \\
\text { on-off }\end{array}$} \\
\hline $1-0$ & 86 & 68 & 84 & 74 & 79 & 75 & 55 & \\
\hline $1-1$ & 83 & & & & & 85 & & \\
\hline $2-0$ & 88 & 71 & 86 & 75 & 89 & 95 & 58 & \\
\hline $1-15$ & 93 & 93 & 92 & 82 & 94 & 89 & 68 & 90 \\
\hline $2-14$ & 92 & 91 & 91 & 92 & 94 & 84 & 62 & \\
\hline $16-0$ & 94 & 87 & 87 & 87 & 95 & 95 & 67 & 90 \\
\hline $1-s$ & 80 & 71 & 81 & 76 & 88 & 82 & 69 & \\
\hline $2-s$ & 76 & 74 & 80 & 72 & 83 & 83 & 61 & \\
\hline $16-s$ & 81 & 73 & 79 & 72 & 88 & 82 & 67 & \\
\hline Aver. $s$ & 79 & 73 & 80 & 73 & 86 & 82 & 66 & \\
\hline Aver. $x-0$ & 89 & 75 & 86 & 79 & 88 & 88 & 60 & \\
\hline
\end{tabular}




\subsection{Temporal factors}

In the tests of the previous sections, the interval between successive display frames was identical to the display duration, and thus these two factors were confounded. If the observer is searching for an unlikely event, e.g., bright 1 's in the first column in the first frame, it is not clear whether the limiting variable is the display duration or the interval between successive displays. Tests were therefore run in which 'on'- and 'off'-frames were interleaved. For example, with 1 on frame and 15 off frames, the interval between successive displays was 16 frames or $458 \mathrm{msec}$, but the duration of presentation was 1 frame or $28.6 \mathrm{msec}$. The left column of tables 3 and 4 list the on-off combinations, in frames, for the display combinations listed on top of the table. In order to obtain comparable ranges of performances among conditions, the probability of a specific parity sum was varied. In these tests, the specific constraints were announced for each trial for the observers. In addition, only the first frame of the sequence of eight frames was presented in single-frame tests marked by $s$ in the lower half of the tables. Also presented is the average score under single-frame presentations, and the average score for 8 -frame sequences with zero off-times. It is noted that under the condition of 1 frame on, 15 frames off, there was a large disturbing flicker, the display appeared to dim, and transitional information supporting good apparent motion was lacking. Since the single-frame tests were not terminated by visual interference, the duration of processing the single frame may have extended far beyond the display duration.

The main result is that, depending upon the specitic condition, performance may be limited either by display duration or by the inter-display time. In most cases, the limiting feature is the inter-display time despite the large flicker in the 1-15 tests. In many instances, performance is also better for the entire sequence of eight successive displays than for the single-frame presentation. Presumably, the informational redundancy associated will successive frames is more powerful than the visual interference associated with successive frames of different items.

\subsection{Effect of 'other' variables}

In the previous tests, when a specific display variable did not enter a given constraint, it was frozen at a given level. For example, $C$ was fixed at character $1, O$ was fixed at an upright orientation, and $B$ was fixed at the highest brightness.

In the tests represented in the left half of table 4 , a weak constraint was imposed upon $C$ and the other variables were either fixed $(F)$, or varied extraneously $(E)$ at random independently of $C$, or slaved $(S)$ to the level of $C$. Extraneous variation of a variable was performed independently of all constraint opcrations. To slave all variables to each other, all variables were represented by the same state, e.g., when state 1 was selected for variable $C$, state 1 was also selected for variables $\phi$ and $B$. The several manipulations were performed under a variety of on-off presentation conditions, as in table 3.

Best performance was achieved with all variables slaved, although the difference between slaving all variables and holding non-constrained variables fixed was small. Poorest performance was achieved by extraneous variation in orientation, or in the simultaneous variation in orientation and brightness.

The tests in the right half of table 4 represent the $C X Y T$ constraint. Under the more complex constraint, the effect of extraneous variables appears to be even larger than under the single variable constraint. 


\section{Discussion}

\subsection{Modes of information processing}

Following Garner and Gottwald (1969), we initially distinguished between two modes of information processing: one is relatively fast, immediately given in experience, in which the individual elements are relatively unanalyzable; the other is relatively slow, given only after considerable effort, in which the individual elements are relatively analyzable. The former may be called 'perceptual', the latter may be called 'cognitive', 'intellectual', or 'verbal'. We may expect the former to be the characteristic mode at rapid rates of presentation, and we may expect the latter to be the characteristic mode at slow rates of processing.

It is tempting to identify improvements in performance associated with slower presentation rates with a 'cognitive' mode of processing. This might have been shown in the present tests, for example, by comparing the growth in performance for $1,2,3$ or more constrained variables as a function of rate of presentation. However, the different baselines of performance make such a comparison difficult.

It is also tempting to speculate that the detection of some welldefined, but infrequent, constraints require extensive visual search, whereas the detection of poorly defined, but frequent, constraints require extended information processing. The latter would presumably be limited by display duration; the latter would presumably be limited by the rate of display presentation. The critical comparison in tables 3 and 4 is between condition $1-15$ and $16-0$. Only a single condition yields a substantial difference in performance in favor of $16-0$, and that involved the $C X Y T$ constraint. Again, the testing of the speculation is hampered by different baselines in performance.

\subsection{Comparison with manipulations of spatial microstructure}

There are many differences between displays employing discrete multi-state numeric characters, and displays encoding binary information in terms of the absence or presence of a dot. Nevertheless, estimates of the depth of information processing - as here defined are comparable in the two cases. For briefly presented displays, only constraints associated with two independent display variables can be 
discerned employing the spatial microstructure of the display with binary-coded elements. In the present tests, better-than-chance, but not excellent, performance can be achieved with combinations of three display variables, presumably because such constraints can be verbalized, e.g. 'search for bright upright 1's.'.

\section{References}

Garner, W. J. and R. L. Gottwald, 1968. The perception and learning of temporal patterns. Quart. Exp. Psychol. 20, 97-109.

Pollack, I., 1971. Detection of one-, two-, and three-dimensional Markov constraints in visual displays. Acta Psychol. 35, 219-232. 